\title{
Gradhiva
}

GRADHIV

Revue d'anthropologie et d'histoire des arts

13 | 2011

Pièges à voir, pièges à penser

\section{Gilles Boëtsch, Dominique Chevé et Hélène Claudot- Hawad (dir.), Décors des corps}

Paris, CNRS éditions, 2010, 398 p.

Jean-Baptiste Eczet

\section{OpenEdition}

Journals

Édition électronique

URL : http://journals.openedition.org/gradhiva/2109

DOI : 10.4000/gradhiva.2109

ISSN : 1760-849X

Éditeur

Musée du quai Branly Jacques Chirac

Édition imprimée

Date de publication : 18 mai 2011

Pagination : 234-235

ISBN : 978-2-35744-042-5

ISSN : 0764-8928

Référence électronique

Jean-Baptiste Eczet, « Gilles Boëtsch, Dominique Chevé et Hélène Claudot-Hawad (dir.), Décors des corps », Gradhiva [En ligne], 13 | 2011, mis en ligne le 18 mai 2011, consulté le 21 septembre 2020. URL : http://journals.openedition.org/gradhiva/2109; DOI : https://doi.org/10.4000/gradhiva.2109

Ce document a été généré automatiquement le 21 septembre 2020.

(c) musée du quai Branly 


\section{Gilles Boëtsch, Dominique Chevé et Hélène Claudot-Hawad (dir.), Décors des corps}

Paris, CNRS éditions, 2010, 398 p.

Jean-Baptiste Eczet

\section{RÉFÉRENCE}

Gilles Boëtsch, Dominique Chevé et Hélène Claudot-Hawad (dir.), Décors des corps. Paris, CNRS éditions, 2010, 398 p.

1 Cet ouvrage intitulé Décors des corps - jeu sur l'homophonie des deux thèmes traités aurait pu s'appeler Couleurs sur corps, du nom de la manifestation qui l'a inspiré, organisée en 2008 par le CNRS. Cette précision permet d'expliquer l'organisation particulière de l'ouvrage: trois leçons inaugurales, suivies de trente-sept courtes études de six à huit pages.

2 Ces études présentent des approches disciplinaires variées - historiques, archéologiques, anthropologiques, sociologiques ou littéraires - et nous ne pourrons pas ici passer en revue la totalité des contributions. Aussi, nous nous contenterons de restituer une impression générale de l'ouvrage après avoir mentionné quelques contributions qui nous ont semblé - et ce ne sont pas les seules - d'un intérêt particulier.

3 Les deux premières « leçons inaugurales » offrent un aperçu utile de ce que la physique et la chimie appellent couleur. Bien que certaines formules mathématiques nous aient échappé, la distinction originelle établie par Goethe entre "couleur-lumière " et «couleur-matière » est exposée selon les canons de formulation des sciences dures. Quelques aperçus historiques, comme la mention de l'émergence de techniques dont le rendu coloré est désormais expliqué par la structure du support (verre rubis, 
iridescence, etc.), décrivent certaines applications et apportent une agréable contextualisation aux lecteurs issus des sciences humaines.

Plusieurs contributions décrivent la construction d'identités complexes au moyen d'usages de décorations et de couleurs sur le corps. Ainsi, Antoine Roullet propose un point de vue original sur les pratiques de mortification des «carmélites déchaussées espagnoles " à partir du xvI siècle. Plutôt que de voir dans la condamnation par le milieu religieux de l'attention portée au corps un rejet pur et simple des cosmétiques, de certaines tenues et de leur corollaire démoniaque (tromperie et déguisement), l'auteur déclare que «le maquillage et la parure "à rebours" de la religieuse sont la manière de rendre visible un réel caché et non plus de cacher le réel» (p.118). Le vêtement de mortification confère à la religieuse qui le porte une identité singulière, car il perd l'uniformité conventionnelle de l'habit religieux en se salissant et en se colorant. Le « discours général et collectif » (p.119) laisse place à une personnalisation des religieuses. L'habit de mortification humilie et constitue également une parodie de l'habit des galantes en exhibant des parties du corps et l'âme pécheresse de la religieuse. De plus, le corps est lui-même "bigarré, rapiécé, blessé par la mortification » (p.119) et constitue une strate plus intime de l'identité qui réplique l'apparence de l'habit : le port du silice colore ainsi le corps du rouge du sang et du noir des plaies. Mais cette mortification sert aussi à révéler, par contraste, la vie et la beauté. La passion du Christ présente en filigrane, l'acte d'humilité et la figuration de la laideur chargent in fine la religieuse d'une intention vertueuse : «La laideur révélée au monde dans un geste d'humilité est le signe le plus sûr d'une beauté cachée, [...] une beauté ravalée à la demande du Seigneur.» (p.122) L'auteur montre bien comment la modification sur les divers couches de l'apparence produit des strates de sens et permet ainsi au corps de figurer l'idéal religieux avec ses ambivalences.

Éric Deroo s'intéresse quant à lui au camouflage militaire et aux peintures de guerre. La description de l'évolution des pratiques de camouflage et de peinture tout au long du $\mathrm{xx}^{\mathrm{e}}$ siècle est à ce sujet tout à fait éclairante. On y apprend que les premiers camouflages ne concernaient que le matériel tandis que les hommes étaient laissés tels quels, probablement en raison de la comparaison, à cette époque peu flatteuse, avec les peintures tribales ou primitives des films hollywoodiens. À l'origine, les artistes chargés de réaliser le camouflage, peintres et sculpteurs, s'inspiraient largement du cubisme et de la dislocation des formes. Puis les premières lignes de camouflages vestimentaires virent le jour, et, à mesure que se perfectionnent les tenues, le camouflage devient l'un des traits distinctifs de certaines troupes, voire d'un ensemble d'unités, la peinture de guerre participant d'une totalisation croissante du camouflage. L'auteur souligne de manière intéressante que, concernant ces pratiques, l'homme est pris entre « la haute technologie, la robotique la plus fine et la plus sophistiquée, et l'environnement dans sa dimension la plus grégaire et brute » et que cela participe de la perte de "quelques dimensions réflexives et morales" (p. 176). On peut toutefois regretter certaines interprétations psychologisantes trop rapides, comme l'idée qu'entre ces deux pôles, il «se naturalise, se déshumanise largement» ou que les peintures de guerre représentent « un moyen de se rassurer » (p. 175). De manière plus générale, le camouflage, en fonction du contexte, sert à se fondre dans un milieu par iconisme ou à s'en détacher de manière visuelle et identitaire (dans le cas des effets de mode), ce qui n'est pas sans rappeler des processus d'évolution de l'apparence animale. Car de tels phénomènes peuvent aboutir à une sorte d'auto-iconisme ${ }^{1}$ où, à ce jeu des 
différences et des ressemblances, on doit tout faire pour être encore plus soi-même. Et l'auteur suggère que la peinture militaire serait le seul attribut exclusif qui permet de différencier un vrai militaire de la «bête de mode » (p. 176).

6 À côté de ces deux études qui décrivent des identités complexes (on pourrait aussi mentionner la contribution de Sylvie Beaud sur les masques de théâtre de Guan Suo au Yunnan), l'ensemble de l'ouvrage brasse des approches et des thématiques bien plus larges. Des traits de peintures sur des statuettes anthropomorphe dans les Cyclades durant le Bronze ancien (Emmanuelle Fournier) aux techniques de bronzage sans soleil (Bernard Andrieu), en passant par le traitement de la couleur dans les vêtements des personnages de Proust (Pierre Zoberman), le cumul des contributions montre l'omniprésence de la couleur sur les corps, bien qu'aucune ligne théorique transversale n'émerge de ces exemples.

7 L'introduction de Gilles Boëtsch, très érudite, postule que «le corps modifié s'arrache au naturel et s'insère dans un système de sens » (p.9). On retrouve cette conception dans la structure même de l'ouvrage : celui-ci débute sur deux textes nous présentant ce qu'est la couleur du point de vue scientifique, "en soi », pour qu'ensuite chaque contribution montre un des usages contextualisés de cette "matière première " universelle, c'est-à-dire par le prisme de la variabilité culturelle que les sciences humaines décrivent. Que le corps soit un donné biologique et que la couleur soit perçue par des récepteurs biologiquement situés ne fait aucun doute, mais on peut s'interroger sur la pertinence à les considérer comme étalon ou point de départ pour l'analyse de comportements humains qui s'élaboreraient de manières diverses à partir d'un donné commun. De nombreux travaux contemporains cherchent en effet à affranchir l'anthropologie et les sciences humaines d'une telle dichotomie'.

8 Une approche générale de la couleur court également le risque de rapprocher des termes qui ne sont pas comparables. Le terme "couleur » ne possède pas d'équivalent dans toutes les langues et sa conceptualisation est très variable. Considérer la couleur en soi revient à sélectionner un certain type de perception visuelle qui renferme déjà sa complexité propre (teinte, saturation, intensité, etc.) et qui, en pratique, n'apparaît que rarement disjointe d'autres qualités, sensibles ou non. Si la couleur semble omniprésente et sature l'espace visuel, elle est cependant toujours incluse dans des matières et des supports autres qu'elle-même (tissus, peintures, cicatrices, tatouages, gemmes, etc.). Annie Mollard-Desfour et Jean Pruvost, deux linguistes-lexicographes, montrent, dans une thèse sur travaux ${ }^{3}$, que définir les mots de couleurs dans un " dictionnaire de langue » se révèle particulièrement délicat : outre la distinction entre la langue courante et la langue de spécialité, c'est la nature descriptive et surtout connotative des mots et expressions de couleurs qui rendent une définition et une traduction difficiles. Ils ajoutent que la couleur traduit des codes culturels et symboliques, qu'elle est un « écho » à autre chose.

9 C'est en fait ce que nous montrent les contributions au volume : bien qu'on puisse la trouver partout, la couleur isolée ne représente plus grand-chose. Contextualisée, elle se dilue dans la variabilité culturelle et dans les divers statuts ontologiques que les hommes lui attribuent, à elle ou à ses supports. Une contribution s'impose sur ce point : Anne Varichon montre que lors du rituel holi en Inde, on utilise de plus en plus des couleurs de synthèse, toxiques pour les organismes, qui permettent une intensification du rendu et sont ainsi plus attractives (la rhodamine B, par exemple, est employée au détriment des colorants traditionnels). Et l'auteur d'ajouter qu'« une large part de 
l'humanité s'est habituée à vivre dans la couleur, toutes les couleurs, même celles réservées jadis aux élites, les plus profondes, les plus éclatantes, les plus subtiles, les plus solides » et que «nous nous sommes mis à exiger la constance de leur éclat et de pouvoir les savourer à moindre coût » (p. 136). N'est-ce pas notre vision scientifique (la couleur comme rayonnement partiellement réfléchi et non comme matière) ainsi que notre technologie (qui permet des niveaux extrêmes de variété et de saturation) qui tendent à autonomiser une perception pour l'instituer ensuite en objet?

10 Si le lecteur ne trouvera pas de proposition générale dans cet ouvrage, tant le corps et la couleur s'avèrent difficiles à conceptualiser, on ne peut toutefois que saluer la volonté de réunir des recherches traitant de ces thèmes importants et passionnants. Ils enrichissent notre compréhension de phénomènes culturels et mettent en avant la fausse évidence de certains objets.

\section{NOTES}

1. Gregory Bateson, Vers une écologie de l'esprit. T. I. Paris, Seuil, 1977.

2. Voir Philippe Descola, Par-delà nature et culture. Paris, Gallimard, 2006 ; et Eduardo Viveiros de Castro, «Le don et le donné, trois nano-essais sur la parenté et la magie », Ethnographiques.org 6, 2004.

3. Annie Mollard-Desfour et Jean Pruvost, Le lexique chromatique français: entre langue courante, langue de spécialité et reflet social : autour de la publication du Dictionnaire des mots et expressions de couleur. Cergy, université de Cergy-Pontoise, thèse de doctorat, 2006.

\section{AUTEURS}

JEAN-BAPTISTE ECZET

jb_eczet@yahoo.fr 